\title{
Arrows of Time and the Anisotropic Properties of CMB
}

\author{
A.E. Allahverdyan ${ }^{1,2)}$, V.G. Gurzadyan ${ }^{2,3)}$ \\ 1) Institute of Theoretical Physics, University of Amsterdam Valckenierstraat 65, 1018 XE Amsterdam, The Netherlands \\ ${ }^{2)}$ Yerevan Physics Institute, Alikhanian Brothers St. 2, Yerevan 375036, Armenia, \\ 3) ICRA, Dipartimento di Fisica, Universita di Roma La Sapienza, 00185 Rome, Italy
}

The relation between the thermodynamical and cosmological arrows of time is usually viewed in the context of the initial conditions of the Universe. It is a necessary but not sufficient condition for ensuring the thermodynamical arrow. We point out that in the Friedmann-Robertson-Walker Universe with negative curvature $k=-1$ there is the second necessary ingredient. It is based on the geodesic mixing the dynamical instability of motion along null geodesics in hyperbolic space. Kolmogorov (algorithmic) complexity as a universal and experimentally measurable concept can be very useful in description of this chaotic behavior using the data on Cosmic Microwave Background radiation. The formulated curvature anthropic principle states the negative curvature as a necessary condition for the time asymmetric Universe with an observer.

PACS: 05.70 Ln, 95.30.-k

\section{INTRODUCTION.}

The Universe is expanding thus determining the cosmological arrow of time. The fate of the expansion, i.e. whether the Universe will expand forever or not, crucially depends on the mean density of the Universe. The Universe is filled by Cosmic Microwave Background radiation (CMB) with Planckian spectrum and highly isotropic temperature.

In the present paper we attempt to argue that these observational facts and the thermodynamical arrow of time, and as the main consequence the second law of thermodynamics, both are the result of the geometry of the Universe, namely, its negative curvature. Thus the fate of the expansion and the second law are linked via the geometry of the Universe.

The association of the second law and the thermodynamical arrow with the geometry of the Universe proposed below differs from other original approaches on the nature of the thermodynamical arrow of Gold [1], Penrose [2] [3], Hawking [4] and Page [5], Prigogine [6], Zurek [7], Zeh [8], among the principal ones.

$\mathrm{CMB}$ is a cornerstone in our discussion. CMB photons are moving freely during the most lifetime of the Universe, thus tracing its geometry. Obviously, CMB have to carry the signature of the geometry. Indeed, the properties of CMB in hyperbolic Friedmann-RobertsonWalker $(k=-1)$ Universe differ from those of flat, $k=0$, and positively curved, $k=+1$, cases. Particularly the exponential deviation of the geodesics and hence, the effect of geodesic mixing in the hyperbolic spaces leads at least to the following observable consequences [9] [10]:

(1) damping of anisotropy after the last scattering epoch;

(2) flattening of autocorrelation function;

(3) distortion of anisotropy spots.

It is remarkable, that the statistically significant signature of the third effect - the threshold independent distortion in the CMB sky maps - has been detected by means of the analysis of COBE DMR 4-year data [11]. If it is really a result of the geodesic mixing, as predicted, then this can be a direct indication of the negative curvature of the Universe, and hence $\Omega_{0}<1$.

It is necessary to note, that at the present many observational facts - particularly on the large-scale distribution of galaxies - are supporting the mean density of the Universe to be below its critical value. There is even an indication on the apparent accelerating rate of the expansion, attributed to the contribution of the cosmological constant (e.g. 12$]$ ).

On the other hand, the time asymmetry of the Universe is one of the deepest and yet challenging problems of modern physics.

Several different and at first glance, independent arrows of time have been defined.

(i) The thermodynamical arrow of time - entropy of a closed system increases with time.

(ii) The cosmological arrow of time - the universe expands.

(iii) The psychological arrow of time - our knowledge about the past is more definite than about the future.

(iv) The arrow of retarded electromagnetic interaction.

Although these arrows arise from CPT-symmetric dynamical laws, they can be associated with CPTasymmetric initial conditions as well; below we deal with only T-invariance, since CP-asymmetry of weak interactions is not directly relevant to the present discussion [2]. The last two arrows are thought as closely related with the thermodynamical arrow [13] 22] 3] [8], so that the main arrows are the cosmological and thermodynamical ones.

Thus in the present paper we claim that if the geometry is basic for the cosmology, it may be equally basic for the thermodynamics. First, we recall that thermodynamical arrow for a closed system can be formulated as a consequence of the following necessary conditions 14 8:

1) Absence of correlations in the initial conditions;

2) Dynamical chaos. 
These two conditions appear already in the Boltzmann's derivation of his famous kinetic equation, though perhaps not explicitly. They can be traced out clearly in Zwanzig's derivation of master-equation [14] or Jaynes' information-theoretical approach to irreversibility 15]. A usual discussion about possible relations between cosmological and thermodynamical arrows of time concentrates only the first condition [2]- [5], [8], [13]. Our purpose here is to point out that the second ingredient of the thermodynamical arrow can have cosmological context as well, and it is ensured due to the mixing of trajectories in hyperbolic spaces. Namely, if the Friedmann-RobertsonWalker universe has negative curvature, then the flow of null geodesics which describes the free motion of photons, represents an Anosov system [16] - a class of dynamical systems with maximally strong statistical properties. Anosov systems are characterized by exponential divergence of initially close trajectories, property of K-mixing, positive KS-entropy, countable Lebesgue spectrum, as well as by the exponential decay of the correlators 17].

On the other hand, intrinsic decoupling of correlations is responsible for the so-called Markovian behavior 19 [21] [23]. As we shall discuss below this latter property can be one of two main ingredients ensuring the thermodynamical arrow [24] [23], though the existence of other mechanisms is not excluded.

Thus we show that the exponential instability of geodesic flow in hyperbolic FRW Universe - the geodesic mixing - revealed through the properties of CMB, is relating the thermodynamical and cosmological arrows of time.

The paper is organized as follows. In section II we discuss the thermodynamical arrow and the conditions which are necessary for its derivation; we follow mainly the arguments of [14] (see also [8])). In section [II] we show how the $\mathrm{CMB}$ mixing properties in the negatively curved space can be connected with the thermodynamical arrow. Then we discuss the algorithmic complexity as a powerful tool for description of the chaotic behavior under discussion (section IV). Our conclusions are represented in the last section.

\section{THERMODYNAMICAL ARROW OF TIME}

Evolution of a closed non-relativistic system (the relativistic case will add only technical difficulties) is described by the Liouville equation for a probability density in the phase space

$$
\mathrm{i} \partial_{t} \rho=[H, \rho] \equiv \mathcal{L} \rho,
$$

where $\mathcal{L}$ is the Liouville operator, $H$ is the Hamiltonian, and $[. ., .$.$] denotes the Poisson bracket as usual. The$ quantum case can be investigated using exactly the same formulae, but Poisson brackets should be substituted by commutators, and probability densities by density matrices. Thus, on the given question, neglecting certain effects [25], there are no principal differences between the classical and quantum approach [14]. The statistical description is started by introducing a set of relevant coarsegrained variables. This means that the exact dynamical equation (11) is projected into a subspace of the phase space. Thus, a linear projection operator $\Pi\left(\Pi=\Pi^{2}\right)$ is introduced, which transforms the density from the full phase space to the subspace of the relevant variables

$$
\Pi \rho=\rho_{r e l},
$$

where $\rho_{\text {rel }}$ is the probability density of the relevant variables. Dividing the initial equation (11) into two parts which correspond to $\rho_{r e l}$ and $\rho_{i r}=\rho-\rho_{r e l}$, we get an equation for $\rho_{\text {rel }}$

$$
\begin{gathered}
\mathrm{i} \partial_{t} \rho_{r e l}=\Pi \mathcal{L} \rho_{\text {rel }} \\
-\mathrm{i} \int_{0}^{t} G(\tau) \rho_{r e l}(t-\tau) \mathrm{d} \tau+\Pi \mathcal{L} \exp (\mathrm{i}(1-\Pi) \mathcal{L} t) \rho_{i r}, \\
\quad G(t)=\Pi \mathcal{L} \exp (-\mathrm{i}(1-\Pi) \mathcal{L} t)(1-\Pi) \mathcal{L} \Pi .
\end{gathered}
$$

Two essential assumptions must be imposed to get the thermodynamical arrow of time in terms of the $\mathrm{H}$ theorem. First, the autonomous character of Eq. (3) should be ensured: $\rho_{\text {rel }}(t)$ can depend only on its initial conditions $\rho_{\text {rel }}(0)$, and not on $\rho_{i r}(0)$. It is necessary to assume that

$$
\rho_{i r}(0)=0,
$$

in order to have self-consistent statistical description. We see that this condition means the absence (in the proper sense) of initial correlations in the system. Conceptually this is very close to the initial conditions of the Universe proposed by Penrose [2] [3]. Ultimately, (5) can be explained simultaneously via cosmological initial conditions [8].

However, even with the condition (5) Eq. (3) does not describe the thermodynamical arrow or the second law. Moreover, it is compatible with an anti-thermodynamical behavior. The second necessary condition concerns the kernel $G(t)$ which arises from the interaction of the relevant degrees of freedom with the irrelevant ones. In other words, this kernel contains the contribution of the entire history of the relevant variables and hence, Eq. (3) is non-Markovian. However, it is by no means trivial that just this fact is incompatible with the thermodynamic arrow 24] 23]. We must have physical conditions supplying the Markovian (no-memory) regime in Eq. (3) 24] [23. Namely, let the kernel $G(t)$ varies much faster than $\rho_{\text {rel }}(t)$; this allows us to consider $\rho_{\text {rel }}$ as constant during the characteristic times of variation of $G(t)$, and hence, to get Markovian, stationary master equation which is compatible with the thermodynamical arrow

$$
\mathrm{i} \partial_{t} \rho_{r e l}=\Pi \mathcal{L} \rho_{r e l}-\mathrm{i} \int_{0}^{\infty} \mathrm{d} \tau G(\tau) \rho_{r e l}(t) .
$$


In the most general form the second law can be expressed by means of decrease of exergy (this term was originally proposed by Clausius)

$$
S(\rho(t)) \leq S\left(\rho\left(t^{\prime}\right)\right) \text { iff } t \geq t^{\prime},
$$

where exergy (also called relative entropy or information gain) is an useful generalization of the concept of thermodynamic potential

$$
S(\rho(t))=\operatorname{tr} \rho\left(\ln \rho-\ln \rho_{s t}\right) .
$$

Here tr means integration by the full phase space in the classical case, and trace in the quantum one. $\rho_{s t}$ is a stationary state attained by the statistical system at very large times. The latter, however, should be understood as still much less than the Poincare recurrent time; the infinity at the upper limit in Eq. (6) should be also considered in this way. Therefore the recurrent time itself must be very large. This condition can be considered as satisfied, since for majority of "reasonable" systems the Poincare time exceeds the age of the Universe. If this stationary distribution is the Gibbs' one, we get free energy from Eq. (8), and for the microcanonical stationary state, describing the closed stat it is reduced to the minus usual Boltzmann-Gibbs-Shannon entropy.

It must be stressed again that the both above mentioned conditions are necessary for the derivation of the thermodynamical arrow. However, it is not the whole story because the second assumption is essentially dynamical, and hence needs a concrete physical mechanism for its validity. One of the possible mechanisms realizing the convergence to Markovian behavior is the intrinsic chaoticity of the considered dynamical system through the effective decoupling of correlators [20] 21] [38]. This property will be discussed in the next section.

At the end of this section let us mention that there are cases where the markovian master equation (6) can appear as too naive. Namely, some additional, essentially system-dependent procedures should be invoked to ensure the correct physical behavior. However, they will not change the physical meaning of the markovian assumption.

\section{GEODESICS MIXING.}

The geodesics of a space (locally if the space is noncompact) with constant negative curvature in all twodimensional directions are known to possess properties of Anosov systems.

As it was proved by Pollicott 17 for $\operatorname{dim}=3$ manifold $M$ with constant negative curvature the time correlation function of the geodesic flow $\left\{f^{\lambda}\right\}$ on the unit tangent bundle $S M$ of $M$

$$
b_{A_{1}, A_{2}}(\lambda)=\int_{S M} A_{1} \circ f^{\lambda} \cdot A_{2} d \mu-\int_{S M} A_{1} d \mu \int_{S M} A_{2} d \mu
$$

is decreasing by exponential law for all functions $A_{1}, A_{2} \in$ $L^{2}(S M)$

$$
\left|b_{A_{1}, A_{2}}(\lambda)\right| \leq c \cdot\left|b_{A_{1}, A_{2}}(0)\right| \cdot e^{-h \lambda},
$$

where $c>0, \mu$ is the Liouville measure and $\mu(S M)=1$, $h$ is the KS-entropy of the geodesic flow $\left\{f^{\lambda}\right\}$.

To reveal the properties of the free motion of photons in pseudo-Riemannian $(3+1)$-space the projection of its geodesics into Riemannian 3-space has to be performed, i.e. by corresponding a geodesic $c(\lambda)=x(\lambda)$ to the geodesic in the former space: $\gamma(\lambda)=(x(\lambda), t(\lambda))$.

Then the transformation of the affine parameter is as follows 18.

$$
\lambda(t)=\int_{t_{0}}^{t} \frac{d s}{a(s)} .
$$

The KS-entropy in the exponential index can be easily estimated for the matter dominated post-scattering Universe [9], so that

$$
e^{h \lambda}=(1+z)^{2}\left[\frac{1+\sqrt{1-\Omega}}{\sqrt{1+z \Omega}+\sqrt{1-\Omega}}\right]^{4} .
$$

i.e. depends on the density parameter $\Omega$ and the redshift of the last scattering epoch $z$. The initial condition (5) should be the one to ensure the thermodynamical arrow.

The decay of correlators for geodesic flow for $k=-1$ FRW Universe provides the procedure of coarse-graining and ensures the Markovian (no-memory) behavior of the CMB parameters (6), so that

$$
t \gg \tau=1 / h,
$$

where KS-entropy defines the characteristic time scale $\tau$ and depends only on the diameter of the Universe (the only scale in the maximally symmetric space) [9]. The time scale $\tau$ is the so-called Markov time or a random variable independent on future as defined in the theory of Markov processes [26], and in CMB problem describes the decay of initial perturbations, i.e. damping of the initial anisotropy amplitude and the flattening of the angular correlation function [9], 10]. For certain dynamical systems that time scale defines also the relaxation time for tending to a microcanonical equilibrium. The evaluation of $\tau$ and hence of the negative curvature of the Universe has been performed in 11] using the COBE data.

The negative constant curvature leads to a decay of time correlators of geodesics, thus defining the thermodynamic arrow for CMB in FRW $k=-1$ Universe.

\section{COMPLEXITY AS A MEASURE OF CHAOTIC BEHAVIOR}

For the description of the chaoticity as an underlying mechanism ensuring the Markovian behavior, which is 
one of the two necessary ingredients of the thermodynamical arrow of time, the algorithmic complexity can appear to be a convenient descriptor.

Algorithmic complexity was introduced by Kolmogorov 27] and independently by Solomonoff and Chaitin [34]. Mathematical aspects of the application of complexity to dynamical systems are discussed in [28]. The notion of algorithmic complexity has been discussed extensively also for physical problems [31] [32] as well. Algorithmic complexity (or algorithmic information) for an object represented by a sequence of bits is defined as the length in bits of the shortest possible program according to which an universal Turing machine (computer), starting from its standard and fixed initial state, produces this object as its single output and halts. The universality of the machine ensures almost machineindependence of the considered definition. As shown the machine-dependent part of the shortest program can be neglected for relatively long sequences [27] 334.

Thus, the definition of the algorithmic complexity for an object $A$ reads

$$
K_{A}=\min _{p}|p(A)|,
$$

where the minimization is made over all programs $p$ computing the object for a fixed universal computer, and $|\ldots|$ denotes the length in bits as usual.

It is natural to call an object complex (or random) if its complexity is comparable with its length [28]. Hence, a chaotic system will possess bigger complexity than a regular one. We are interested in specific complexity [28]

$$
k(A)=\frac{K_{A}}{|A|},
$$

so that a finite non-zero $k(A)$ will mean that the object $A$ is algorithmically complex. As shown random sequences are indistinguishable (for all practical purposes) from the ones generated by the proper stochastic process [29]. Notice that the time of computation does not enter in the definition of complexity; this is the first hint that the exact calculation of complexity can be hopeless. Moreover, it was proved [29] that complexity is not a computable quantity. In fact, it is necessary for its self-consistent definition. In order to define complexity for a dynamical system one should consider a translation of its trajectory into a symbolic language [28] [19]. Namely, the phase space is divided into non-overlapping regions, and every such a region gets its symbol. Then a trajectory of the considered dynamical system can be viewed as a sequence of symbols (merely as a matter of convenience this sequence can be translated into the language of bits). Now the dynamics can be called chaotic (for fixed initial conditions) if the corresponding symbolic sequence is algorithmically complex [28] [30]. Note that the above mentioned partition should be detailed enough because algorithmic complexity is well-defined only for sufficiently long sequences of symbols.
Besides the natural form of this definition, it has welldefined advantages compared to the more traditional one through Lyapunov exponents. Indeed, the traditional signatures of chaos are less obvious if the attention is shifted from the time evolution of the phase-space points to the time evolution of probability densities, governed by the coarse-grained Liouville equation. Namely, if the distance between two densities is defined in terms of an overlap integral, there is no exponential divergence of initially close densities 32. On the other hand, needless to say that trajectories and small shifting of initial conditions cannot be available in many systems of practical interest. It is the main reason of a recent activity seeking unambiguous relations between KS-entropy and directly measurable quantities like excess of entropy [35]. On the other hand, the definition through algorithmic complexity is already given in the terms of coarse-grained description which is reasonable also in the general context of the thermodynamical arrow of time.

The fundamental theorem of Brudno and Alekseev 28] states, that if KS-entropy is non-zero for a dynamical system, then there is the specific algorithmic complexity. It is also natural that in a random sequence there are no long-range correlations. Such a correlation will introduce some "order", and lead to a decrease of complexity. Thus, the definition through algorithmic complexity allows to illustrate effectively this point also.

In the case of the CMB problem, the convenience of use of the complexity is determined by two reasons. First, one can derive a relation between the curvature of the Universe and the complexity of the CMB anisotropy spots [36], and, second, a concrete algorithm can be created enabling to calculate the complexity of the spots at the CMB sky maps [37]. The algorithm developed in [37] showed that the complexity is indeed a calculable quantity for digitized CMB data, and its values are well correlating with the values of the fractal dimension of the spots, and therefore the complexity can be a powerful descriptor for the analysis of the data of forthcoming CMB experiments. Other algorithms, particularly based on the random sequence concepts, may be developed as well.

Thus the complexity allows to illustrate effectively the chaos-negative curvature link through the CMB properties.

\section{CONCLUSION}

Thus, the negative curvature of the FRW Universe and the effect of geodesic mixing can provide the condition necessary for the emergence of the thermodynamic arrow of time. Moreover this mechanism can explain why CMB contains the major fraction of the entropy of the Universe 39.

If this is indeed the mechanism of the origin of the thermodynamic arrow, then the thermodynamics in a flat and 
positively curved universes not necessarily to be strongly time asymmetric, and the latter is observed since we happened to live in a Universe with negative curvature. In other words, the symmetry of the Newtonian mechanics, electrodynamics, quantum mechanics might purely survive in some universes. On the other hand, a recent activity devoted to the foundations of thermodynamics 40] allows to disentangle time-asymmetric elements from the remained basis. We are going to consider separately the relations of the above-mentioned theory with the possible thermodynamics description arising in the flat or negatively curved space 25].

In this context the essence of thermodynamical arrow must be understood as not the mere increase of entropy of an almost closed system, but the fact that this arrow has the universal direction in the entire Universe (see [41]). In the light of our suggested explanation of the emergence of this arrow, it follows that the negative curvature is the very mechanism unifying all local thermodynamical arrows. While in the flat or positively curved universes, i.e. at the absence of a global unification mechanism, there can be local thermodynamical arrows with various directions.

Another intriguing problem arising here, is whether life can occur in such globally time symmetric universes, or the time asymmetry/negative curvature is a necessary ingredient for developing of life - curvature anthropic principle. CMB features have to carry the signature of this principle.

We thank Th.M. Nieuwenhuizen and W. Zurek for valuable discussions.

[1] T. Gold, Am. J. Phys. 30, 403, (1962).

[2] R. Penrose, in General Relativity: An Einstein Centenary Survey ed. S.W. Hawking, W. Israel, Cambridge University Press, Cambridge, (1979).

[3] R. Penrose, The Emperor's New Mind, Oxford University Press, 1989; J. Stat.Phys. 77, 217, (1994).

[4] S.W. Hawking, Phys. Rev. D, 32, 2989, (1985).

[5] D. Page, Phys. Rev. D, 32, 2496, (1985).

[6] T.Petrosky, I.Prigogine, Advances in Chemical Physics, XCIX, 1, (1997).

[7] W. Zurek, in Physical Origins of Time Asymmetry, ed. J.J. Halliwell, J. Perez-Mercader, W.H. Zurek, p.175, Cambridge University Press, (1996).

[8] H.D. Zeh, The Physical Basis of the Direction of Time, Springer, Berlin, (1992); C. Kiefer, H.D. Zeh, Phys. Rev. D, 51, 4145, (1995).

[9] V.G.Gurzadyan, A.A.Kocharyan, Astr.Astrophys., 260, 14,(1992); Int. Journ. Mod. Phys. D. 2, 97, (1993); Europhys.Lett. 22, 231, (1993); in Quantum Gravity VI, ed. V.A. Berezin, V.A. Rubakov, D.V. Semikoz, p. 542, World Scientific, (1998).

[10] V.G. Gurzadyan, A.A. Kocharyan, Paradigms of the
Large-Scale Universe, Gordon and Breach Publ., New York, (1994).

[11] V.G. Gurzadyan, S. Torres, Astr.Astrophys. 321, 19, (1997).

[12] P.J.E.Peebles, Nature, 398, 25, (1999).

[13] P.C.W. Davies The Physics of Time Asymmetry, University of California Press, Berkeley, (1976).

[14] R. Zwanzig, J. Chem. Phys., 33, 1338, (1960).

[15] E. T. Jaynes, Phys. Rev. 108, 171, (1957); in Papers on Probability, Statistics, and Statistical Physics, ed. R. D. Rosenkrantz, Kluwer, Dordrecht, (1983).

[16] D.V. Anosov, Comm. Steklov Math.Inst., 90, 1, (1967).

[17] M. Pollicott, J.Stat.Phys., 67, 667, (1992).

[18] C.M. Lockhart, B. Misra, I. Prigogine, Phys.Rev., D25, 921, (1982).

[19] V. I. Arnold and A. Avez, Ergodic Problems of Classical Mechanics, Benjamin, New York, (1968).

[20] M.C. Mackay, Rev. Mod. Phys., 61, 981, (1989).

[21] G.M. Zaslavski, R.Z. Sagdeev, Introduction to Nonlinear Physics, Nauka, Moscow, (in Russian), (1988).

[22] W.J. Cocke, Phys. Rev., 160, 1165, (1967).

[23] A.E. Allahverdyan, D.B. Saakian, Phys.Rev. E, 58, 1148, (1998).

[24] F. Shlogl, Phys. Rep.,62, 268, (1980).

[25] A.E. Allahverdyan, V.G. Gurzadyan, in preparation.

[26] E.B.Dynkin, Markov Processes, Springer-Verlag, New York, (1965).

[27] A.N. Kolmogorov, Probl.Inform.Transmis., 1, 3, (1965).

[28] V. M. Alekseev, M. V. Yakobson, Phys. Rep. 75, 287, (1981).

[29] A.K. Zvonkin, L.A. Levin, UMN, XXV, 85 (1970).

[30] J. Ford, Phys. Today, 36(4), 40, (1983).

[31] W. H. Zurek, Phys. Rev. A 40, 4731, (1989).

[32] C. M. Caves, Phys. Rev. E 47, 4010, (1993).

[33] S. Lloyd, Phys. Rev. A 39, 5378, (1989).

[34] G. J. Chaitin, Algorithmic Information Theory, Cambridge University Press, Cambridge, (1987).

[35] M. Dzugutov, et al. Phys. Rev. Lett., 81, 1762, (1998); M. Dzugutov, Nature, 381, 137, (1996).

[36] V.G. Gurzadyan, Europhys. Lett., 46, 114, (1999).

[37] A.E. Allahverdyan, V.G. Gurzadyan, and A.A. Soghoyan Int. Jour. Mod. Phys. D 8, 1, (1999).

[38] C. Jarzynski, Phys. Rev. Lett., 74, 2937, (1995).

[39] A.E. Allahverdyan, V.G. Gurzadyan, Th.M. Nieuwenhuizen, in preparation.

[40] E.H. Lieb, J. Yngvason, Phys. Rep., 310, 1, (1999).

[41] M. Gell-Mann, J.B. Hartle, in Physical Origins of Time Asymmetry, ed. J.J. Halliwell, J. Perez-Mercader, W.H. Zurek, p.311, Cambridge University Press, (1996). 